\title{
Photon-photon correlations as a probe of vacuum-induced coherence effects
}

\author{
Sumanta Das and G. S. Agarwal \\ Department of Physics, Oklahoma State University, Stillwater, Oklahoma 74078, USA
}

(Received 12 November 2007; published 27 March 2008)

\begin{abstract}
We show how the effects of vacuum-induced coherence can be realized by studying photon-photon correlation in the $\pi$-polarized fluorescence in the $j=1 / 2$ to $j=1 / 2$ transition. These effects should thus be observable in measurements of photon statistics in, for example, $\mathrm{Hg}$ and $\mathrm{Ba}$ ion traps.
\end{abstract}

DOI: 10.1103/PhysRevA.77.033850

PACS number(s): 42.50.Ar, 42.50.Dv, 42.50.Lc

\section{INTRODUCTION}

An early work [1] predicted an unusual effect of quantum interference in the problem of spontaneous emission. It was shown that in a degenerate V-shaped system one could get population trapping and generation of quantum coherences in the excited states. This comes about due to the interference between different channels of spontaneous emission. One of the key conditions for the occurrence was that the dipole matrix elements of the two transitions from the excited states to the common ground state were nonorthogonal. Meanwhile, a very large body of theoretical literature has been devoted to the subject of vacuum-induced coherences [2-9] and a nice review is given in Ref. [2]. It was also suggested how the above condition on dipole matrix elements can be bypassed if we consider an anisotropic vacuum [10] which, for example, would be the case when considering emission from excited atoms or nanoparticles [11]. We thus look for possible realistic systems where vacuum-induced coherences (VICs) are observable. Kiffner et al. [12] showed that an atomic system with degenerate transitions $j=1 / 2 \leftrightarrow j=1 / 2$ would be a suitable system where effects of VICs are prominent. They showed how vacuum-induced coherences change the spectrum of the emitted radiation. While the results of Kiffner $e t a l$. for the spectrum are quite interesting, much of the current experimental effort [13-16] is focused on the study of photon-photon correlations [17]. Thus one would like to understand if the vacuum-induced coherences significantly affect the photon-photon correlations. This is the question we examine. The significance of VICs depends on the system under consideration. We deal specifically with the $j$ $=1 / 2$ to $j=1 / 2$ transitions, the details of which are given in the next section, where we also explain how VICs are important for such a system. We mention explicitly two systems where we have such transitions; thus these systems would, for example, be candidates for the effects of VICs on photonphoton correlations. These systems are (A) a single ${ }^{198} \mathrm{Hg}^{+}$ ion in a trap [13] and (B) a single ${ }^{138} \mathrm{Ba}^{+}$ion in a trap [14]. In both cases the ground level is $6 s^{2} S_{1 / 2}$ and the excited level is $6 p^{2} P_{1 / 2}$. The measurement of photon-photon correlation for such multilevel systems can be done in the standard way (see Diedrich et al. $[17,14]$ ).

It may be added that the photon-photon correlations have acquired new significance in the context of quantuminformation processing and quantum imaging as well as in interferences from independent atoms $[15,16,18]$. Thus it is pertinent to check if VIC effects are to be included in the calculation of photon-photon correlations for a given experimental system. The organization of this paper is as follows. In Sec. II we introduce the model and present the working equations. In Sec. III we calculate the photon-photon correlations both in the presence and in the absence of vacuuminduced interference effects. In Sec. IV we present numerical results to highlight the effects of vacuum-induced coherences on photon-photon correlations. In Sec. V we conclude with the outlook and future directions.

\section{MODEL}

Figure 1 shows the level scheme of a four-level atom modeled with a $j=1 / 2$ to $j=1 / 2$ transition. This kind of level scheme is realizable, for example, in ${ }^{198} \mathrm{Hg}^{+}[13]$ and ${ }^{138} \mathrm{Ba}^{+}$ [14] ions. The ground level is $6 s^{2} S_{1 / 2}$ and the excited level is $6 p{ }^{2} P_{1 / 2}$. Each of these levels is twofold degenerate. We exhibit the magnetic sublevels explicitly. The nature of transitions between these levels can be found from various dipole matrix elements. The dipole matrix elements can be obtained from the Wigner-Eckart theorem and the Clebsch-Gordan coefficients. They are found to be

$$
\begin{gathered}
\vec{d}_{31}=-\vec{d}_{42}=-\frac{1}{\sqrt{6}} \mathcal{D} \hat{e}_{z}, \\
\vec{d}_{41}=\vec{d}_{32}^{*}=\frac{1}{\sqrt{3}} \mathcal{D} \hat{\epsilon}_{-},
\end{gathered}
$$

with $\hat{\epsilon}_{-}=(\hat{x}-i \hat{y}) / \sqrt{2}$ and $\hat{e}_{z}$ the unit vector along the $z$ direction. In Eq. (1) $\mathcal{D}$ denotes the reduced matrix element of the dipole moment operator $\vec{d}$. Thus the transitions $|1\rangle \leftrightarrow|4\rangle$ and $|2\rangle \leftrightarrow|3\rangle$ couple to $\sigma^{+}$- and $\sigma^{-}$-polarized light, respectively.

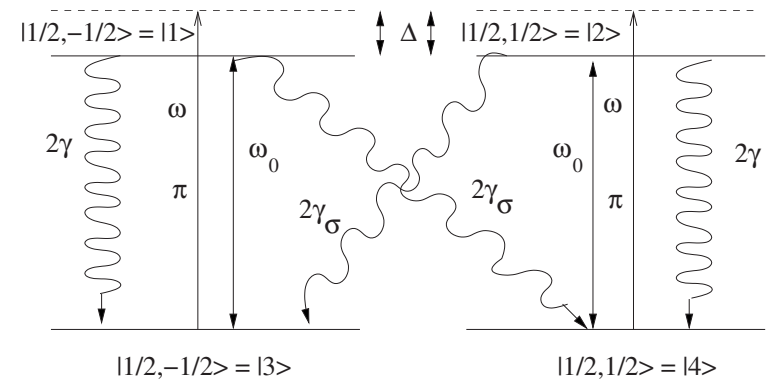

FIG. 1. Schematic diagram of a four-level atom modeled by the $j=1 / 2$ to $j=1 / 2$ transition. 
The transitions $|1\rangle \leftrightarrow|3\rangle$ and $|2\rangle \leftrightarrow|4\rangle$ couple to light linearly polarized along $\hat{e}_{z}$. The spontaneous decays of the excited state to the two ground states are given by $2 \gamma$ and $2 \gamma_{\sigma}$ as shown in the figure. The spontaneously emitted photons are $\pi$ polarized in the transitions $|1\rangle \leftrightarrow|3\rangle,|2\rangle \leftrightarrow|4\rangle$ whereas they are $\sigma$ polarized in the transitions $|1\rangle \leftrightarrow|4\rangle,|2\rangle \leftrightarrow|3\rangle$. The four-level system is driven by a $\pi$-polarized monochromatic field of frequency $\omega$,

$$
\vec{E}(t)=\mathcal{E}_{0} e^{-i \omega t} \hat{e}_{z}+\text { c.c. },
$$

where c.c. is the complex conjugate. With this particular choice of polarization, the driving field couples only to the two transitions $|1\rangle \leftrightarrow|3\rangle$ and $|2\rangle \leftrightarrow|4\rangle$. The total Hamiltonian for this atom-field system is given by

$$
\mathcal{H}=\mathcal{H}_{A}+\mathcal{H}_{I},
$$

where the unperturbed Hamiltonian for the atom is

$$
\mathcal{H}_{A}=\hbar \sum_{i=1}^{2} \omega_{0}|i\rangle\langle i|,
$$

where energies are measured from the ground level. The interaction Hamiltonian is given by

$$
\mathcal{H}_{I}=-\vec{d} \cdot \vec{E}(t)=\hbar \Omega(|1\rangle\langle 3|-| 2\rangle\langle 4|) e^{-i \omega t}+\text { H.c. },
$$

where H.c. is the Hermitian conjugate and $\Omega$ is the Rabi frequency defined by

$$
\Omega=\mathcal{E}_{0} \frac{\vec{d}_{42} \cdot \hat{e}_{z}}{\hbar}=\frac{1}{\sqrt{6}} \mathcal{D} \mathcal{E}_{0} / \hbar .
$$

The time evolution of this four-level system is investigated by studying the density matrix equation. The spontaneous emission is included via master equation techniques. Following the standard procedure [1], we obtain

$$
\begin{gathered}
\dot{\rho}=-\frac{i}{\hbar}[\mathcal{H}, \rho]+\mathcal{L} \rho, \\
\mathcal{L} \rho=-\gamma_{\sigma}\left(|1\rangle\langle 1|\rho+| 2\rangle\langle 2|\rho+\rho| 1\rangle\langle 1|+\rho| 2\rangle\langle 2|-2| 3\rangle\langle 3| \rho_{22}\right. \\
\left.-2|4\rangle\langle 4| \rho_{11}\right)-\gamma(|1\rangle\langle 1|\rho+| 2\rangle\langle 2|\rho+\rho| 1\rangle\langle 1|+\rho| 2\rangle\langle 2| \\
\left.-2|3\rangle\left\langle 3\left|\rho_{11}-2\right| 4\right\rangle\langle 4| \rho_{22}\right)+\gamma\left(|4\rangle\left\langle 3\left|\rho_{21}+\right| 3\right\rangle\langle 4| \rho_{12}\right),
\end{gathered}
$$

The last two terms in Eq. (7) arise from the vacuum-induced interference and it comes because the dipole matrix elements $\vec{d}_{13}$ and $\vec{d}_{24}$ are antiparallel. In a frame rotating with the frequency of the coherent drive, the density matrix equations are

$$
\begin{gathered}
\dot{\tilde{\rho}}_{11}=i \Omega^{*} \widetilde{\rho}_{13}-i \Omega \widetilde{\rho}_{31}-2 \Gamma \widetilde{\rho}_{11}, \\
\dot{\tilde{\rho}}_{22}=i \Omega \widetilde{\rho}_{42}-i \Omega^{*} \widetilde{\rho}_{24}-2 \Gamma \widetilde{\rho}_{22}, \\
\dot{\tilde{\rho}}_{33}=i \Omega \widetilde{\rho}_{31}-i \Omega^{*} \widetilde{\rho}_{13}+2 \gamma_{\sigma} \widetilde{\rho}_{22}+2 \gamma \widetilde{\rho}_{11}, \\
\dot{\tilde{\rho}}_{12}=-i \Omega \widetilde{\rho}_{32}-i \Omega^{*} \widetilde{\rho}_{14}-2 \Gamma \widetilde{\rho}_{12},
\end{gathered}
$$

$$
\begin{gathered}
\dot{\tilde{\rho}}_{13}=-i \Delta \widetilde{\rho}_{13}+i \Omega\left(\widetilde{\rho}_{11}-\widetilde{\rho}_{33}\right)-\Gamma \widetilde{\rho}_{13}, \\
\dot{\tilde{\rho}}_{14}=-i \Delta \widetilde{\rho}_{14}-i \Omega \widetilde{\rho}_{12}-i \Omega \widetilde{\rho}_{34}-\Gamma \widetilde{\rho}_{14}, \\
\dot{\tilde{\rho}}_{23}=-i \Delta \widetilde{\rho}_{23}+i \Omega \widetilde{\rho}_{21}+i \Omega \widetilde{\rho}_{43}-\Gamma \widetilde{\rho}_{23}, \\
\dot{\tilde{\rho}}_{24}=-i \Delta \widetilde{\rho}_{24}-i \Omega\left(\widetilde{\rho}_{22}-\widetilde{\rho}_{44}\right)-\Gamma \widetilde{\rho}_{24}, \\
\dot{\tilde{\rho}}_{34}=-i \Omega \widetilde{\rho}_{32}-i \Omega^{*} \widetilde{\rho}_{14}-\gamma \widetilde{\rho}_{12},
\end{gathered}
$$

where

$$
\begin{gathered}
\tilde{\rho}_{i i}=\rho_{i i}, \quad \tilde{\rho}_{12}=\rho_{12}, \quad \tilde{\rho}_{34}=\rho_{34}, \\
\tilde{\rho}_{i j}=\rho_{i j} e^{-i \omega t} \quad(i=1,2 ; j=3,4), \\
\Gamma=\left(\gamma_{\sigma}+\gamma\right), \quad \Delta=\omega-\omega_{13}=\omega-\omega_{24} .
\end{gathered}
$$

The remaining equations can be generated by taking complex conjugates and using $\operatorname{Tr}(\rho)=1$. The steady-state solution of Eq. (8) is found to be

$$
\begin{gathered}
\tilde{\rho}_{12}=\tilde{\rho}_{14}=\tilde{\rho}_{32}=\widetilde{\rho}_{34}=0, \\
\tilde{\rho}_{11}=\tilde{\rho}_{22}=\frac{1}{2} \frac{|\Omega|^{2}}{\left(2|\Omega|^{2}+\Gamma^{2}+\Delta^{2}\right)}, \\
\tilde{\rho}_{33}=\tilde{\rho}_{44}=\frac{1}{2} \frac{|\Omega|^{2}+\Gamma^{2}+\Delta^{2}}{\left(2|\Omega|^{2}+\Gamma^{2}+\Delta^{2}\right)}, \\
\tilde{\rho}_{13}=-\tilde{\rho}_{24}=-\frac{i \Omega}{\Gamma+i \Delta}\left(\frac{1}{2} \frac{\Gamma^{2}+\Delta^{2}}{\left(2|\Omega|^{2}+\Gamma^{2}+\Delta^{2}\right)}\right) .
\end{gathered}
$$

As can be seen from Eqs. (10) and (11) the vacuum-induced interference has no effect on the steady-state solutions. Thus one should investigate how vacuum-induced coherences can show up in dynamical quantities like the correlation functions.

\section{PHOTON-PHOTON CORRELATIONS}

Since the objective of this paper is to investigate the observable consequences of vacuum-induced coherence, we focus our attention on the photon statistics of the radiation emitted by our model system. In particular, we will calculate photon-photon correlations as currently considerable experimental effort is focused on such correlations. For this we need to know how to relate the atomic properties to the statistical properties of the spontaneously emitted radiation. The answer to this question already exists in quantum theory. In fact, from the existing literature [19], we know that the positive frequency part of the electric field operator at a point $\vec{r}$ in the far-field zone can be written in terms of the atomic operators as 


$$
\begin{aligned}
\vec{E}^{+}(\vec{r}, t)= & \vec{E}_{0}^{+}(\vec{r}, t)-k_{0}^{2} \sum_{i}\left\{\left[\hat{R}_{i} \times\left(\hat{R}_{i} \times \vec{d}_{31}\right)|3\rangle\left\langle\left. 1\right|_{\tau}\right]\right.\right. \\
& +\left[\hat{R}_{i} \times\left(\hat{R}_{i} \times \vec{d}_{42}\right)|4\rangle\left\langle\left. 2\right|_{\tau}\right]+\left[\hat{R}_{i} \times\left(\hat{R}_{i} \times \vec{d}_{32}\right)|3\rangle\left\langle\left. 2\right|_{\tau}\right]\right.\right. \\
& +\left[\hat{R}_{i} \times\left(\hat{R}_{i} \times \vec{d}_{41}\right)|4\rangle\left\langle\left. 1\right|_{\tau}\right]\right\} R_{i}^{-1} e^{-i\left(k_{0} \hat{r} \cdot \vec{r}_{i}+\omega \tau\right)},
\end{aligned}
$$

where $\vec{R}_{i}=\vec{r}-\vec{r}_{i}, \vec{r}$ being the distance of the point of observation from the origin and $\vec{r}_{i}$ being the position of the atom from the origin. Further, $\tau=t-\frac{r}{c}$ is the retarded time, $k_{0}=\frac{\omega_{0}}{c}$, $\omega_{0}=\omega_{13}=\omega_{24}$, and $\vec{d}_{i j}$ is the electric dipole moment operator. The first term on the right of Eq. (12) is the free-field term and the second term is the retarded dipole field emitted by the atom. The emitted radiation consists of different polarization components-the $\pi$ - and the $\sigma$-polarized components. In Eq. (12) the terms $|3\rangle\left\langle\left. 1\right|_{\tau}\right.$ and $\left.\mid 4\right\rangle\left\langle\left. 2\right|_{\tau}\right.$ correspond to $\pi$ polarization whereas $|3\rangle\left\langle\left. 2\right|_{\tau}\right.$ and $\left.\mid 4\right\rangle\left\langle\left. 1\right|_{\tau}\right.$ correspond to $\sigma$ polarization. We next calculate the photon-photon correlations and the normalized second-order correlations for the emitted radiation from the $\pi$ transitions of this driven fourlevel atom. For $\pi$ polarization the relevant part of the electric field operator is given by

$$
\begin{aligned}
\vec{E}^{+}(\vec{r}, t)= & \vec{E}_{0}^{+}(\vec{r}, t)-\left(\frac{\omega_{0}}{c}\right)^{2} \frac{1}{r}\left\{[ \hat { n } \times ( \hat { n } \times \vec { d } _ { 3 1 } ) ] | 3 \rangle \left\langle\left.1\right|_{\tau}\right.\right. \\
& +\left[\hat{n} \times\left(\hat{n} \times \vec{d}_{42}\right)\right]|4\rangle\left\langle\left. 2\right|_{\tau}\right\},
\end{aligned}
$$

where $\tau$ as before is the retarded time $t-r / c$. In the lowestorder correlation the free-field term of Eq. (13) does not contribute. This can be seen directly from the definition of quantized fields [19], the fact that the field is initially in the vacuum state, and the expression for the normally ordered correlation function for the field, $\left\langle\vec{E}^{-}(\vec{r}, t) \cdot \vec{E}^{+}\left(\vec{r}^{\prime}, t^{\prime}\right)\right\rangle$. Hence, with no contribution from the free-field term the intensity $I_{\pi}$ of the light emitted in the $\pi$ transition from the atom is

$$
\begin{aligned}
\left\langle I_{\pi}\right\rangle= & \left\langle\vec{E}_{\pi}^{-}(\vec{r}, t) \cdot \vec{E}_{\pi}^{+}(\vec{r}, t)\right\rangle \\
= & \left(\frac{\omega_{0}}{c}\right)^{4} \frac{1}{r^{2}}\left\langle\left[\hat{n} \times\left(\hat{n} \times \vec{d}_{31}\right)\right]^{*} \cdot\left[\hat{n} \times\left(\hat{n} \times \vec{d}_{31}\right)\right] \mid 1\right\rangle\left\langle\left. 1\right|_{\tau}\right. \\
& +\left[\hat{n} \times\left(\hat{n} \times \vec{d}_{42}\right)\right]^{*} \cdot\left[\hat{n} \times\left(\hat{n} \times \vec{d}_{42}\right)\right]|2\rangle\left\langle\left. 2\right|_{\tau}\right\rangle,
\end{aligned}
$$

where we have taken our origin at the location of the atom, $\vec{r}=\hat{n} r, \tau$ is the retarded time, and we used the property $A_{i j} A_{k l}=A_{i l} \delta_{k j}$. The negative frequency part of the electric field operator $\vec{E}^{-}(\vec{r}, t)$ can be found by taking the complex conjugate of the positive frequency part. Now, if we assume that the point of observation lies perpendicular to both the polarization and propagation directions, we have from Eq. (14)

$$
\left\langle I_{\pi}\right\rangle=\left(\frac{\omega_{0}}{c}\right)^{4} \frac{1}{r^{2}}\left(\left|\vec{d}_{31}\right|^{2}\langle\mid 1\rangle\langle 1 \mid\rangle_{\tau}+\left|\vec{d}_{42}\right|^{2}\langle\mid 2\rangle\langle 2 \mid\rangle_{\tau}\right) .
$$

Equation (15) can be further simplified using Eqs. (1) and (11), where in using Eq. (11) we have assumed that observation is being made at the long-time limit. The final expression for $I_{\pi}$ in the long-time limit (steady state) is then

$$
\left\langle I_{\pi}\right\rangle^{s t}=\left(\frac{\omega_{0}}{c}\right)^{4} \frac{|\mathcal{D}|^{2}}{6 r^{2}} \frac{|\Omega|^{2}}{\left(2|\Omega|^{2}+\Gamma^{2}+\Delta^{2}\right)} .
$$

Equation (16) clearly show that intensity emitted in the $\pi$ transitions is not altered by vacuum-induced coherences and is simply proportional to the steady-state population of the excited states.

Let us now investigate what happens in the case of twotime photon-photon correlations in the $\pi$ transitions. The two-time photon-photon correlation for the level scheme in Fig. 1 can be written as

$$
\begin{aligned}
\left\langle I_{\pi}(t+\tau) I_{\pi}(t)\right\rangle= & \left\langle\vec{E}_{\pi}^{-}(\vec{r}, t) \vec{E}_{\pi}^{-}(\vec{r}, t+\tau): \vec{E}_{\pi}^{+}(\vec{r}, t+\tau) \vec{E}_{\pi}^{+}(\vec{r}, t)\right\rangle \\
= & \left(\frac{\omega_{0}}{c}\right)^{8} \frac{1}{r^{4}}\left\{\left[\hat{n} \times\left(\hat{n} \times \vec{d}_{31}\right)\right]^{*} \cdot[\hat{n} \times(\hat{n}\right. \\
& \left.\left.\left.\times \vec{d}_{31}\right)\right]\right\}^{2}\left\langle(|1\rangle\langle 3|-| 2\rangle\langle 4|)_{t}(|1\rangle\langle 1|+| 2\rangle\right. \\
& \left.\times\langle 2|)_{t+\tau}(|3\rangle\langle 1|-| 4\rangle\langle 2|)_{t}\right\rangle,
\end{aligned}
$$

The two-time correlation function that appears in Eq. (17) is to be obtained from the solution of the time-dependent density matrix equations [Eq. (9)] and the quantum regression theorem [20]. In the rest of the paper we deal with correlations like (17) in the steady state, where these depend only on the time difference $\tau$ and thus the retarded time becomes irrelevant. A closer look at Eq. (9) shows that eight of the fifteen equations form a closed set of linear equations which can be solved to find $|1\rangle\left\langle\left. 1\right|_{t+\tau}, \mid 2\right\rangle\left\langle\left. 2\right|_{t+\tau}\right.$ and hence the term $(|1\rangle\langle 1|+| 2\rangle\langle 2|)_{t+\tau}$ in Eq. (17). Before going further let us list those eight equations:

$$
\begin{gathered}
\dot{\tilde{\rho}}_{11}=i \Omega^{*} \widetilde{\rho}_{13}-i \Omega \widetilde{\rho}_{31}-2 \Gamma \widetilde{\rho}_{11}, \\
\dot{\tilde{\rho}}_{33}=i \Omega \widetilde{\rho}_{31}-i \Omega^{*} \widetilde{\rho}_{13}+2 \gamma_{\sigma} \widetilde{\rho}_{22}+2 \gamma \widetilde{\rho}_{11}, \\
\dot{\tilde{\rho}}_{13}=-i \Delta \widetilde{\rho}_{13}+i \Omega\left(\widetilde{\rho}_{11}-\widetilde{\rho}_{33}\right)-\Gamma \widetilde{\rho}_{13}, \\
\dot{\tilde{\rho}}_{31}=i \Delta \widetilde{\rho}_{31}-i \Omega^{*}\left(\widetilde{\rho}_{11}-\widetilde{\rho}_{33}\right)-\Gamma \widetilde{\rho}_{31}, \\
\dot{\tilde{\rho}}_{22}=i \Omega \widetilde{\rho}_{42}-i \Omega^{*} \widetilde{\rho}_{24}-2 \Gamma \widetilde{\rho}_{22} \\
\dot{\tilde{\rho}}_{24}=i \Omega^{*} \widetilde{\rho}_{24}-i \Omega \widetilde{\rho}_{42}+\gamma_{\sigma} \widetilde{\rho}_{11}+\gamma \widetilde{\rho}_{22} \\
\dot{\tilde{\rho}}_{42}=i \Delta \widetilde{\rho}_{24}-i \Omega\left(\widetilde{\rho}_{22}-i \widetilde{\rho}_{44}\right)-\Gamma \widetilde{\rho}_{24},
\end{gathered}
$$

In compact notation these equations can be written as

$$
\dot{\tilde{\rho}}=\mathcal{M} \tilde{\rho},
$$

where $\dot{\tilde{\rho}}$ and $\tilde{\rho}$ are $(8 \times 1)$ column matrices and $\mathcal{M}$ is an $(8$ $\times 8)$ square matrix. Now, using the method depicted in [21] and using Eq. (18), we can express the solution of $\langle\mid 1\rangle\left\langle\left. 1\right|_{t+\tau}\right\rangle$ and $\langle\mid 2\rangle\left\langle\left. 2\right|_{t+\tau}\right\rangle$ in the form 


$$
\begin{aligned}
\langle\mid 1\rangle\left\langle\left. 1\right|_{t+\tau}\right\rangle= & f_{11}(\tau)\langle\mid 1\rangle\left\langle\left. 1\right|_{t}\right\rangle+f_{12}(\tau)\langle\mid 3\rangle\left\langle\left. 3\right|_{t}\right\rangle+f_{13}(\tau)\langle\mid 3\rangle\left\langle\left. 1\right|_{t}\right\rangle \\
& +f_{14}(\tau)\langle\mid 1\rangle\left\langle\left. 3\right|_{t}\right\rangle+f_{15}(\tau)\langle\mid 2\rangle\left\langle\left. 2\right|_{t}\right\rangle+f_{16}(\tau)\langle\mid 4\rangle\left\langle\left. 4\right|_{t}\right\rangle \\
& +f_{17}(\tau)\langle\mid 4\rangle\left\langle\left. 2\right|_{t}\right\rangle+f_{18}(\tau)\langle\mid 2\rangle\left\langle\left. 4\right|_{t}\right\rangle, \\
\langle\mid 2\rangle\left\langle\left. 2\right|_{t+\tau}\right\rangle= & f_{51}(\tau)\langle\mid 1\rangle\left\langle\left. 1\right|_{t}\right\rangle+f_{52}(\tau)\langle\mid 3\rangle\left\langle\left. 3\right|_{t}\right\rangle+f_{53}(\tau)\langle\mid 3\rangle\left\langle\left. 1\right|_{t}\right\rangle \\
& +f_{54}(\tau)\langle\mid 1\rangle\left\langle\left. 3\right|_{t}\right\rangle+f_{55}(\tau)\langle\mid 2\rangle\left\langle\left. 2\right|_{t}\right\rangle+f_{56}(\tau)\langle\mid 4\rangle\left\langle\left. 4\right|_{t}\right\rangle \\
& +f_{57}(\tau)\langle\mid 4\rangle\left\langle\left. 2\right|_{t}\right\rangle+f_{58}(\tau)\langle\mid 2\rangle\left\langle\left. 4\right|_{t}\right\rangle,
\end{aligned}
$$

where the $f$ 's are defined by

$$
f_{i k}(\tau)=\left(e^{\mathcal{M} \tau}\right)_{i k},
$$

and

$$
\begin{gathered}
\mathcal{M}_{i k}=\sum_{l} P_{i l} \Lambda_{l l} P_{l k}^{-1}, \\
\left(e^{\mathcal{M} \tau}\right)_{i k}=\sum_{l} P_{i l} e^{\Lambda_{l l} \tau} P_{l k}^{-1} .
\end{gathered}
$$

Here we have diagonalized the matrix $\mathcal{M}$, with $\Lambda$ being the eigenvalues and $P$ the corresponding eigenvectors. We now make use of the quantum regression theorem to obtain the two-time correlation function as

$$
\begin{aligned}
\left\langle B^{\dagger}(t)(|1\rangle\langle 1|+| 2\rangle\langle 2|)_{t+\tau} B(t)\right\rangle= & F_{1}(\tau)\left\langle B^{\dagger}(t) \mid 1\right\rangle\left\langle\left. 1\right|_{t} B(t)\right\rangle \\
& +F_{2}(\tau)\left\langle B^{\dagger}(t) \mid 3\right\rangle\left\langle\left. 3\right|_{t} B(t)\right\rangle \\
& +F_{3}(\tau)\left\langle B^{\dagger}(t) \mid 3\right\rangle\left\langle\left. 1\right|_{t} B(t)\right\rangle \\
& +F_{4}(\tau)\left\langle B^{\dagger}(t) \mid 1\right\rangle\left\langle\left. 3\right|_{t} B(t)\right\rangle \\
& +F_{5}(\tau)\left\langle B^{\dagger}(t) \mid 2\right\rangle\left\langle\left. 2\right|_{t} B(t)\right\rangle \\
& +F_{6}(\tau)\left\langle B^{\dagger}(t) \mid 4\right\rangle\left\langle\left. 4\right|_{t} B(t)\right\rangle \\
& +F_{7}(\tau)\left\langle B^{\dagger}(t) \mid 4\right\rangle\left\langle\left. 2\right|_{t} B(t)\right\rangle \\
& +F_{8}(\tau)\left\langle B^{\dagger}(t) \mid 2\right\rangle\left\langle\left. 4\right|_{t} B(t)\right\rangle,
\end{aligned}
$$

where we define the operator $B$ as $B^{\dagger}(t)=(|1\rangle\langle 3|-| 2\rangle\langle 4|)_{t}$; $B(t)=\left[B^{\dagger}(t)\right]^{\dagger}$ and $F_{i}(\tau)=f_{1 i}(\tau)+f_{5 i}(\tau)$. Using this definition of the operator in Eq. (17), the expression for the two-time photon-photon correlation becomes

$$
\begin{aligned}
\left\langle I_{\pi}(t+\tau) I_{\pi}(t)\right\rangle= & \left(\frac{\omega_{0}}{c}\right)^{8} \frac{1}{r^{4}}\left\{\left[\hat{n} \times\left(\hat{n} \times \vec{d}_{31}\right)\right]^{*} \cdot[\hat{n} \times(\hat{n}\right. \\
& \left.\left.\left.\times \vec{d}_{31}\right)\right]\right\}^{2}\left\langle B^{\dagger}(t)(|1\rangle\langle 1|+| 2\rangle\langle 2|)_{t+\tau} B(t)\right\rangle,
\end{aligned}
$$

which, when Eq. (24) is used, simplifies to

$$
\begin{aligned}
\left\langle I_{\pi}(t+\tau) I_{\pi}(t)\right\rangle= & \left(\frac{\omega_{0}}{c}\right)^{8} \frac{1}{r^{4}}\left\{\left[\hat{n} \times\left(\hat{n} \times \vec{d}_{31}\right)\right]^{*} \cdot[\hat{n} \times(\hat{n}\right. \\
& \left.\left.\left.\times \vec{d}_{31}\right)\right]\right\}^{2}\left[F_{2}(\tau)\langle\mid 1\rangle\langle 1 \mid\rangle_{t}+F_{6}(\tau)\langle\mid 2\rangle\langle 2 \mid\rangle_{t}\right] .
\end{aligned}
$$

In the long-time limit, $\langle\mid 1\rangle\langle 1 \mid\rangle_{t} \equiv \widetilde{\rho}_{11}(t)$ and $\langle\mid 2\rangle\langle 2 \mid\rangle_{t} \equiv \widetilde{\rho}_{22}(t)$, where $\widetilde{\rho}_{11}(t), \tilde{\rho}_{22}(t)$ are the steady-state populations of the excited states given by Eq. (11). Now, following our assump- tion that the point of observation lies perpendicular to both the polarization and propagation directions and substituting for $\widetilde{\rho}_{11}$ and $\tilde{\rho}_{22}$ from Eq. (11), we can simplify Eq. (26) further. The final expression for the two-time photon-photon correlation is then

$$
\begin{aligned}
\mathrm{G}_{\pi}^{(2)}(\tau) & =\left\langle I_{\pi}(t+\tau) I_{\pi}(t)\right\rangle \\
& =\left(\frac{\omega_{0}}{c}\right)^{8} \frac{|\mathcal{D}|^{4}}{36 r^{4}}\left[F_{2}(\tau)+F_{6}(\tau)\right]\left(\frac{1}{2} \frac{|\Omega|^{2}}{\left(2|\Omega|^{2}+\Gamma^{2}+\Delta^{2}\right)}\right),
\end{aligned}
$$

where we have used Eq. (1) for the dipole matrix elements. Note that $F_{2}(\tau)\left[F_{6}(\tau)\right]$ is the sum of probabilities of finding the atom in the states $|1\rangle$ and $|2\rangle$ given that, at $\tau=0$, the atom was in the state $|3\rangle(|4\rangle)$. In the limit of large $\tau$,

$$
\mathrm{G}_{\pi}^{(2)}(\tau) \rightarrow\left(\frac{\omega_{0}}{c}\right)^{8} \frac{|\mathcal{D}|^{4}}{36 r^{4}}\left(\frac{2|\Omega|^{2}}{\left(2|\Omega|^{2}+\Gamma^{2}+\Delta^{2}\right)}\right) .
$$

Next let us derive the expression for two-time photon-photon correlation in the absence of interference. In this case the total photon-photon correlation will be a simple addition of photon-photon correlations for radiation emitted in individual $\pi$ transitions:

$$
\begin{aligned}
\mathbf{G}_{\pi}^{(2)}(\tau)= & \left\langle I_{\pi}(t+\tau) I_{\pi}(t)\right\rangle \\
= & \left\langle\vec{E}_{\pi}^{-}(\vec{r}, t) \vec{E}_{\pi}^{-}(\vec{r}, t+\tau): \vec{E}_{\pi}^{+}(\vec{r}, t+\tau) \vec{E}_{\pi}^{+}(\vec{r}, t)\right\rangle_{|1\rangle\langle 3|} \\
& +\left\langle\vec{E}_{\pi}^{+}(\vec{r}, t+\tau) \vec{E}_{\pi}^{+}(\vec{r}, t) \vec{E}_{\pi}^{+}(\vec{r}, t+\tau) \vec{E}_{\pi}^{+}(\vec{r}, t)\right\rangle_{|2\rangle\langle 4|}, \\
= & \left(\frac{\omega_{0}}{c}\right)^{8} \frac{1}{r^{4}}\left\{\left[\hat{n} \times\left(\hat{n} \times \vec{d}_{31}\right)\right]^{*} \cdot[\hat{n} \times(\hat{n}\right. \\
& \left.\left.\left.\times \vec{d}_{31}\right)\right]\right\}^{2}\langle\mid 1\rangle\left\langle\left. 3\right|_{t}(|1\rangle\langle 1|)_{t+\tau} \mid 3\right\rangle\left\langle\left. 1\right|_{t}\right\rangle \\
& +\left(\frac{\omega_{0}}{c}\right)^{8} \frac{1}{r^{4}}\left\{\left[\hat{n} \times\left(\hat{n} \times \vec{d}_{42}\right)\right]^{*} \cdot\left[\hat{n} \times\left(\hat{n} \times \vec{d}_{42}\right)\right]\right\}^{2} \\
& \times\langle\mid 2\rangle\left\langle\left. 4\right|_{t}(|2\rangle\langle 2|)_{t+\tau} \mid 4\right\rangle\left\langle\left. 2\right|_{t}\right\rangle .
\end{aligned}
$$

Finally, using Eqs. (20), (21), and (11) we get the photonphoton correlation in the absence of interference as

$$
\mathbf{G}_{\pi}^{(2)}(\tau)=\left(\frac{\omega_{0}}{c}\right)^{8} \frac{|\mathcal{D}|^{4}}{36 r^{4}}\left[f_{12}(\tau)+f_{56}(\tau)\right]\left(\frac{1}{2} \frac{|\Omega|^{2}}{\left(2|\Omega|^{2}+\Gamma^{2}+\Delta^{2}\right)}\right) .
$$

Here $f_{12}(\tau)\left[f_{56}(\tau)\right]$ is the probability of finding the atom in the state $|1\rangle(|2\rangle)$ given that, at $\tau=0$, the atom was in the state $|3\rangle(|4\rangle)$. Equation (31) in the limit of large $\tau$ becomes

$$
\mathbf{G}_{\pi}^{(2)}(\tau) \rightarrow\left(\frac{\omega_{0}}{c}\right)^{8} \frac{|\mathcal{D}|^{4}}{36 r^{4}}\left(\frac{|\Omega|^{2}}{\left(2|\Omega|^{2}+\Gamma^{2}+\Delta^{2}\right)}\right) .
$$

We now further calculate the normalized photon-photon correlation corresponding to Eqs. (27) and (31). The $g^{(2)}$ function gives the nonclassical aspects of photon statistics, 
TABLE I. Eigenvalues (in units of $\gamma_{0}$ ) for the diagonalized matrix $\mathcal{M}$ corresponding to two different values of the Rabi frequency of the driving field which is on resonance with the atomic transitions.

\begin{tabular}{ccc}
\hline \hline$\lambda / \gamma_{0}$ & $\Omega=0.5 \gamma_{0}$ & $\Omega=3.0 \gamma_{0}$ \\
\hline 1 & $(-0.349797,-1.10904)$ & $(-0.375000,5.99870)$ \\
2 & $(-0.349797,1.10904)$ & $(-0.375000,-5.99870)$ \\
3 & $(-0.215794,-1.09726)$ & $(-0.208269,5.99522)$ \\
4 & $(-0.215794,1.09726)$ & $(-0.208269,-5.99522)$ \\
5 & $(-0.300406,0.000000)$ & $(-0.250000,0.000000)$ \\
6 & $(-0.165314,0.000000)$ & $(-0.250000,0.000000)$ \\
7 & $(-0.403098,0.000000)$ & $(-0.333462,0.000000)$ \\
8 & $(0.000000,0.000000)$ & $(0.000000,0.000000)$ \\
\hline \hline
\end{tabular}

$$
g^{(2)}(t+\tau, t)=\frac{\left\langle I_{\pi}(t+\tau) I_{\pi}(t)\right\rangle}{\left\langle I_{\pi}(t+\tau)\right\rangle\left\langle I_{\pi}(t)\right\rangle}=\frac{\left[F_{2}(\tau)+F_{6}(\tau)\right] \widetilde{\rho}_{11}}{4 \widetilde{\rho}_{11}^{2}}
$$

$$
\begin{aligned}
\mathbf{g}^{(2)}(t+\tau, t) & =\frac{\left\langle I_{\pi}(t+\tau) I_{\pi}(t)\right\rangle}{\left[\left\langle I_{\pi}(t+\tau)\right\rangle\left\langle I_{\pi}(t)\right\rangle\right]_{|1\rangle\langle 3|}+\left[\left\langle I_{\pi}(t+\tau)\right\rangle\left\langle I_{\pi}(t)\right\rangle\right]_{|2\rangle\langle 4|}} \\
& =\frac{\left[f_{12}(\tau)+f_{56}(\tau)\right] \widetilde{\rho}_{11}}{2 \widetilde{\rho}_{11}^{2}} .
\end{aligned}
$$

Here $\widetilde{\rho}_{11}$ is the steady-state population of the excited state given by Eq. (11) and $g^{(2)}\left(\mathbf{g}^{(2)}\right)$ is the normalized two-time photon-photon correlation function corresponding to the presence (absence) of vacuum-induced interference.

\section{NUMERICAL RESULTS}

In this section we present our numerical results and discuss their consequences. To begin with, we first discuss our method of computation. The decay rates of the excited states to the two ground states, $2 \gamma_{\sigma}$ and $2 \gamma$, are proportional to

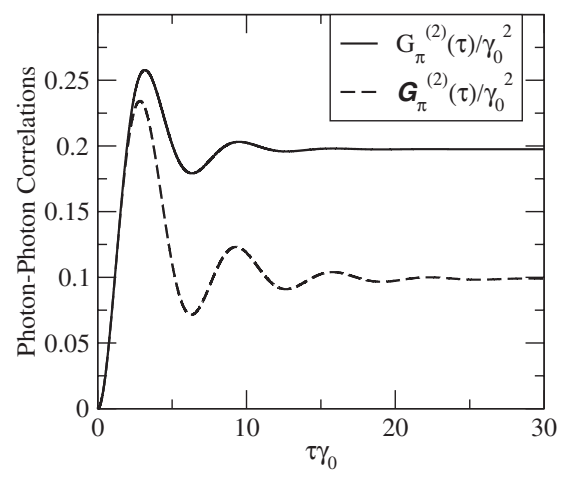

FIG. 2. Plot of two-time photon-photon correlation as a function of time for $\Omega=0.5 \gamma_{0}, \Delta=0.0 \gamma_{0}$, where $\gamma_{0}=4|\mathcal{D}|^{2} \omega_{14}^{3} / 3 c^{3}$. All the plotted parameters are normalized with respect to $\gamma_{0}$ rendering them dimensionless. The solid and dashed lines in this figure and Figs. 3, 4, and 6 correspond to photon-photon correlations in the presence and absence of VICs, respectively.

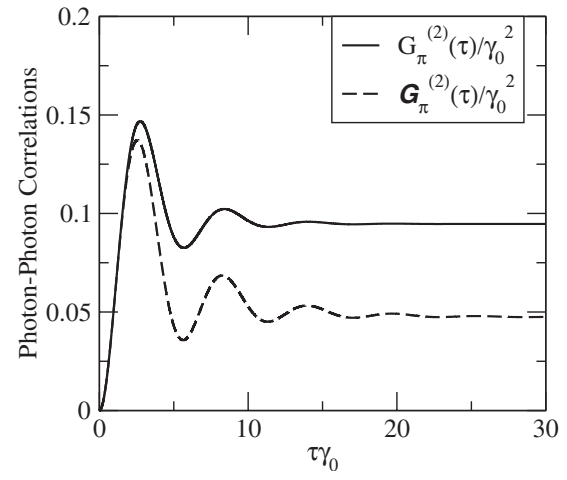

FIG. 3. Plot of two-time photon-photon correlation as a function of time but now for a small detuning $\Delta=0.5 \gamma_{0}$; other parameters remain the same as in Fig. 2.

$\left|\vec{d}_{41}\right|^{2}$ and $\left|\vec{d}_{31}\right|^{2}$, respectively. From Eq. (1) we get $2 \gamma_{\sigma}$ $\equiv \gamma_{0} / 3$ and $2 \gamma \equiv \gamma_{0} / 6$, where $\gamma_{0}$ is proportional to the square of the reduced dipole matrix element. We use these values for the decays in our numerical computation and normalize all the computational parameters with respect to $\gamma_{0}$. Further, we use standard subroutines to diagonalize the complex general matrix $\mathcal{M}$ and obtain complex eigenvalues and eigenvectors of the form $(\alpha+i \beta)$. For all values of detuning and Rabi frequency used in our computation, we have two pairs of complex conjugate eigenvalues, and four other eigenvalues whose complex parts are so small compared to the real parts that these complex parts have no significant contributions. Hence these four eigenvalues can be taken to be purely real. Note that this is in contrast to the case of photon-photon correlations for the two-level model where the number of eigenvalues is 4 [22]. The changes in the eigenvalues lead to spectral modification as discussed by Kiffner et al. [12]. The eigenvalues for $\Omega_{c}=0.5 \gamma_{0}$, and $\Omega_{c}=3 \gamma_{0}$ and detuning $\Delta=0$ are listed in Table I. Note, for example, that for $\Omega_{c}=3 \gamma_{0}$ we have eigenvalues $\pm 5.99870 i-0.375$ and $\pm 5.99522 i$ -0.208269 . This difference in the real parts can produce a dip in the sidebands in the Mollow spectrum [23]. Next we calculate the elements $f_{i j}$ of the $8 \times 8$ matrix $[f]$ using Eqs. (22) and (23). Finally we use the elements $f_{i j}$ corresponding

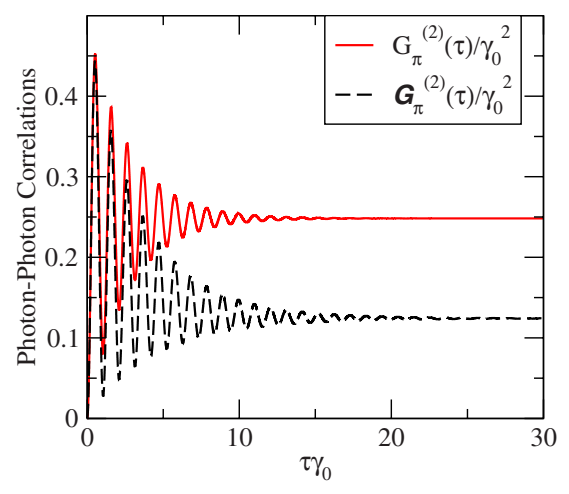

FIG. 4. (Color online) Plot of two-time photon-photon correlation as a function of time for $\Omega=3.0 \gamma_{0}, \Delta=0.0 \gamma_{0}$, where $\gamma_{0}$ $=4|\mathcal{D}|^{2} \omega_{14}^{3} / 3 c^{3}$. All the plotted parameters are dimensionless. The solid red line in this figure corresponds to photon-photon correlation in the presence of VICs. 


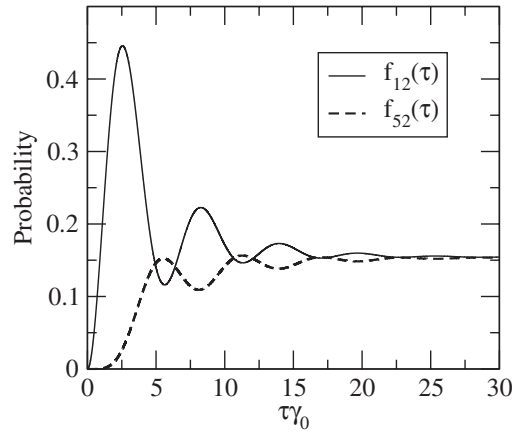

FIG. 5. Probability for finding the atom in states $|1\rangle\left(f_{12}\right)$ and $|2\rangle$ $\left(f_{52}\right)$ given that at time $\tau=0$ the atom was in the state $|3\rangle$ for $\Omega$ $=0.5 \gamma_{0}, \Delta=0.5 \gamma_{0}$, where $\gamma_{0}=4|\mathcal{D}|^{2} \omega_{14}^{3} / 3 c^{3}$. All the plotted parameters are dimensionless.

to Eqs. (27) and (31) and Eqs. (33) and (34) to evaluate the two-time photon-photon correlations and normalized photonphoton correlations in the presence and absence of vacuuminduced interference, respectively.

Figures 2-4 show photon-photon correlations corresponding to Eqs. (27) and (31). The solid and dashed lines in the figures correspond, respectively, to photon-photon correlations in the presence and absence of interference. The correlations calculated in the presence of interference show strong damping of the oscillations and attain an overall higher value as the time separation $\tau$ between two counts increases. The differences between $G^{(2)}$ and $\mathbf{G}^{(2)}$ are most noticeable in the limit of large time separation $\tau$. In order to understand this, we examine the distinction between $F_{2}(\tau)=f_{12}(\tau)+f_{52}(\tau)$ and $f_{12}(\tau)$. We recall that $f_{12}\left(f_{52}\right)$ was the probability of finding the atom in the state $|1\rangle(|2\rangle)$ given that at $\tau=0$ it was in the state $|3\rangle$. We exhibit these probabilities in Fig. 5. We observe that the function $f_{52}(\tau)$ starts becoming significant at a time scale of the order of $\gamma_{\sigma}^{-1}$.

Further, for large $\tau, f_{12}$ and $f_{52}$ become comparable. The physical process that contributes to $f_{52}$ is the following:

$$
|3\rangle \underset{\pi \text { pol }}{\rightarrow}|1\rangle \underset{\text { emission }}{\rightarrow}|4\rangle \underset{\pi \text { pol }}{\rightarrow}|2\rangle .
$$

Similarly, population can start from the state $|4\rangle$ and end up in the state $|1\rangle$ via

$$
|4\rangle \underset{\pi \text { pol }}{\rightarrow}|2\rangle \underset{\text { emission }}{\rightarrow}|3\rangle \underset{\pi \text { pol }}{\rightarrow}|1\rangle .
$$

We show normalized photon-photon correlations in a typical case in Fig. 6. In the case of interference, we observe stron-

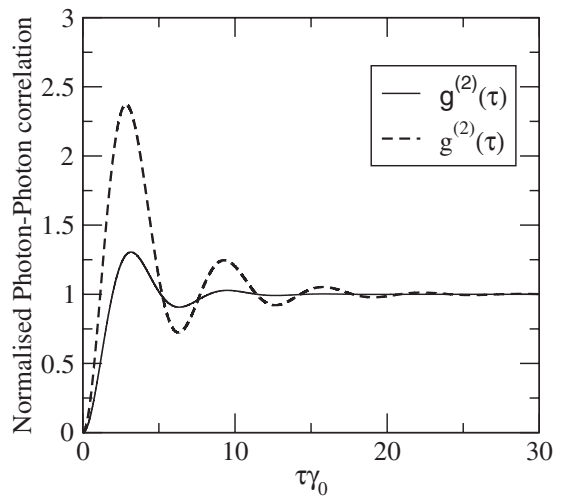

FIG. 6. Normalized photon-photon correlations plotted as a function of time for $\Omega=0.5 \gamma_{0}, \Delta=0.0 \gamma_{0}$, where $\gamma_{0}=4|\mathcal{D}|^{2} \omega_{14}^{3} / 3 c^{3}$. All the plotted parameters are dimensionless.

ger damping of the oscillations and an overall reduction of the $g^{(2)}$ function at shorter time scales. At long-time limits $g^{(2)}(\tau \rightarrow \infty)$ is 1 . The photon antibunching effect is also visible as $0 \leq g^{(2)}(0)<1$. For a shorter time scale we get $g^{(2)}(\tau) \not 1$, a clear signature of the nonclassical nature of the two-time photon-photon correlations.

\section{CONCLUSIONS}

In conclusion, we have shown that the vacuum-induced coherences do significantly affect the two-time photonphoton correlations even though they show no effect on the total steady-state intensity of the radiation emitted in the $\pi$ transitions. The effect of this coherence is reflected in the form of stronger damping and overall larger values of the correlation function $G^{(2)}$. The level scheme $j=1 / 2 \rightarrow j=1 / 2$ is easily realizable and has already been used, for example, in ${ }^{198} \mathrm{Hg}^{+}$[13] in the context of interferences produced by a system of two ions and more recently in ${ }^{138} \mathrm{Ba}^{+}[14]$ in the context of emission in the presence of a mirror. In future we hope to investigate how the asymmetry in the level structures introduced by a magnetic field [24] would influence the photon-photon correlations. This might in turn give us more freedom in choosing the level structure and hence a broader choice in selecting atomic transitions for experiments. Finally, note that it would also be interesting to examine the VIC effects in the context of nonlinear optical effects using $j=1 / 2$ to $j=1 / 2$ transitions.

\section{ACKNOWLEDGMENTS}

This work was supported by NSF Grant No. PHY0665393
[1] G. S. Agarwal, Quantum Statistical Theories of Spontaneous Emission and Their Relation to Other Approaches, Springer Tracts in Modern Physics: Quantum Optics (Springer-Verlag, Berlin, 1974), p. 94.

[2] Z. Ficek and S. Swain, J. Mod. Opt. 49, 3 (2002).
[3] M. Macovei and C. H. Keitel, Phys. Rev. Lett. 91, 123601 (2003); J. Evers and C. H. Keitel, ibid. 89, 163601 (2002); C. H. Keitel, ibid. 83, 1307 (1999).

[4] E. Paspalakis and P. L. Knight, Phys. Rev. A 63, 065802 (2001); E. Paspalakis, N. J. Kylstra, and P. L. Knight, Phys. 
Rev. Lett. 82, 2079 (1999).

[5] Z. Ficek and S. Swain, Phys. Rev. A 69, 023401 (2004); P. Zhou and S. Swain, Phys. Rev. Lett. 77, 3995 (1996).

[6] G. S. Agarwal and A. K. Patnaik, Phys. Rev. A 63, 043805 (2001); S. Menon and G. S. Agarwal, ibid. 57, 4014 (1998).

[7] K. T. Kapale, M. O. Scully, S. Y. Zhu, and M. S. Zubairy, Phys. Rev. A 67, 023804 (2003); O. Kocharovskaya, A. B. Matsko, and Y. Rostovtsev, ibid. 65, 013803 (2001); J. Javanainen, Europhys. Lett. 17, 407 (1992).

[8] Recent works by M. O. Scully; A. Svidzinsky, H. Lipkin, and M. O. Scully, Laser Phys. 17, 635 (2007); Fermi's Golden Rule does not adequately describe Dicke's Supperradiance: A. Svidzinsky, H. Lipkin and M. O. Scully (to be published); M. O. Scully and R. J. Glauber (to be published), this papers examine the role of VICs in the context of the Dicke problem.

[9] It may be noticed that other terms like spontaneously generated coherence and vacuum-induced quantum interferences have been used in the literature.

[10] G. S. Agarwal, Phys. Rev. Lett. 84, 5500 (2000).

[11] A. Rahmani, C. Patrick Chaumet, and Frèdèrique de Fornel, Phys. Rev. A 63, 023819 (2001).

[12] M. Kiffner, J. Evers, and C. H. Keitel, Phys. Rev. Lett. 96, 100403 (2006); Phys. Rev. A 73, 063814 (2006).

[13] U. Eichmann, J. C. Bergquist, J. J. Bollinger, J. M. Gilligan, W. M. Itano, D. J. Wineland, and M. G. Raizen, Phys. Rev. Lett. 70, 2359 (1993).

[14] U. Dorner and P. Zoller, Phys. Rev. A 66, 023816 (2002);
François Dubin, Daniel Rotter, Manas Mukherjee, Carlos Russo, Jürgen Eschner, and Rainer Blatt, Phys. Rev. Lett. 98, 183003 (2007). Following the earlier theoretical work of Dorner and Zoller this experiment examined photon correlation effects in the presence of a mirror, whereas we are specifically studying the effects of VICs in the emission in free space.

[15] T. Chaneliere, D. N. Matsukevich, S. D. Jenkins, S.-Y. Lan, R. Zhao, T. A. B. Kennedy, and A. Kuzmich, Phys. Rev. Lett. 98, 113602 (2007).

[16] P. Maunz, D. L. Moehring, S. Olmschenk, K. C. Younge, D. N. Matsukevich, and C. Monroe, Nat. Phys. 3, 538 (2007).

[17] H. J. Kimble, M. Dagenais, and L. Mandel, Phys. Rev. Lett. 39, 691 (1977); F. Diedrich and H. Walther, ibid. 58, 203 (1987).

[18] C. Thiel, T. Bastin, J. Martin, E. Solano, J. von Zanthier, and G. S. Agarwal, Phys. Rev. Lett. 99, 133603 (2007).

[19] G. S. Agarwal, Quantum Statistical Theories of Spontaneous Emission and Their Relation to Other Approaches (Ref. [1]), pp. 39-40.

[20] M. Lax, Phys. Rev. 172, 350 (1968).

[21] G. S. Agarwal, Phys. Rev. A 15, 814 (1977).

[22] H. J. Carmichael and D. F. Walls, J. Phys. B 9, L43 (1976).

[23] B. R. Mollow, Phys. Rev. 188, 1969 (1969).

[24] S. Menon and G. S. Agarwal [Laser Phys. 9, 813 (1999)] show how a nondegenerate four-level system can produce a modulated response due to VICs. 